\title{
A Five-Year Experience of Sparing Treatment of Descending Aortic Atherosclerotic Aneurysms
}

\author{
Vladimir Petrovich Krylov, Alexander Gennadyevich Mrochek, \\ Valentina Nukolaevna Gayduk, Leonid Ivanovich Reut, Aleksey Leonidovich Smaliakou, \\ Nadezhda Vladimirovna Mankevich, Roman Nikolaevich Pizhik \\ Republican Research and Practical Centre "Cardiology", Ministry of Health of the Republic of Belarus, Minsk, \\ Belarus \\ Email: fun-69@yandex.ru
}

Received 5 February 2016; accepted 12 April 2016; published 15 April 2016

Copyright (C) 2016 by authors and Scientific Research Publishing Inc.

This work is licensed under the Creative Commons Attribution International License (CC BY). http://creativecommons.org/licenses/by/4.0/

(c) (i) Open Access

\begin{abstract}
A growing number of specialists are now beginning to ascertain that treatment of individuals with descending aortic atherosclerotic aneurysms must be provided by cardiologists on a scheduled basis. Surgery is feasible when there is a risk of aneurysm rupture. It requires for the development of conservative treatments and elaboration of indications for surgery. A total of 97 patients with thoracic aortic atherosclerotic aneurysms (TAAA) and abdominal aortic aneurysms (AAA) have been examined over a 5 -year period. They received multifaceted anti-inflammatory medical treatment to strengthen the aortic wall and control its possible expansion. Operative treatment was offered only if there was a risk of aneurysm rupture. One of the principal factors adversely affecting mortality is the presence of co-morbidities requiring permanent medical corrective treatment irrespective of surgical or medical treatment provided. It is also important to outline the indications for surgery based on multifactorial pathogenetic manifestations. Treatment aiming at the reversal of ethiopathogenic mechanisms of disease progression contributes to a significant longer survival in DAA patients.
\end{abstract}

\section{Keywords}

Aortic Atherosclerotic Aneurysms, Surgical Treatment, Conservative Treatment, Outcomes

\section{Introduction}

Assuming the similarity of ethiopathogenetic processes occurring in the aortic wall, the present study combines

How to cite this paper: Krylov, V.P., Mrochek, A.G., Gayduk, V.N., Reut, L.I., Smaliakou, A.L., Mankevich, N.V. and Pizhik, R.N. (2016) A Five-Year Experience of Sparing Treatment of Descending Aortic Atherosclerotic Aneurysms. World Journal of Cardiovascular Surgery, 6, 47-54. http://dx.doi.org/10.4236/wjcs.2016.64009 
the terms of descending thoracic and abdominal aortic segments into "descending aortic segments" and uses the term "descending aortic aneurysms" (DAA).

Aortic aneurysms may develop in any segment. Twenty-five percent of all aneurysms are descending thoracic aortic aneurysms (DTAA). Other aneurysms (75\%) form in the abdominal segment [1]-[4]. Four to seven percent of patients show the involvement of descending thoracic and abdominal aorta (thoracic-abdominal aneurysms). When no treatment, in $75 \%$ of patients death occurs at 3 years [5] [6], and only $46 \%$ at 5 years.

Causes of death are diverse, but $35 \%$ - $40 \%$ of patients die due to aneurysm rupture, about one-third die due to heart failure, and $14 \%$ - $20 \%$ due to pulmonary complications and pneumonia [7]-[9]. In the presence of small aneurysms with no clinical signs, a dynamic follow-up including US and CT examinations every 6 months to assess aneurysm condition is recommended. Aneurysm enlargement by more than $5 \mathrm{~mm}$ at 6 months or apparent pain syndrome implicates surgical treatment [4].

In small-sized abdominal aortic aneurysms, one-year survival rate is $75 \%$, while it is only $48 \%$ at 5 years. If aneurysm diameter is above $6 \mathrm{~cm}$, 1 -year survival becomes $50 \%$, and it is only $6 \%$ at 5 years [10]. Based on the available data, there is no significant difference in the presentation of thoracic or abdominal aneurysm disease, while technically, surgery performed on descending thoracic aorta may be challenging compared to infrarenal site [3] [11].

Natural history of DAA is not fully understood. Traditionally, it is considered that the growth of aneurysm diameter is inevitable with the rupture as a natural outcome. However, some patients with small DAA may show stabilization of the disease [3] [11].

Despite improvements in surgical techniques, the outcomes have not improved. There is a wide debate in literature sources concerning both management and treatment of patients, and ethiopathogenesis of the disease [4] [11]-[15]. Scientific community is searching for a cause of still high mortality. It is due to aneurysm rupture, concomitant multi-organ dysfunction and over indications for surgery [4] [8] [16] [17]. But the main thing is that the signs of fatal outcome cannot be predicted in the majority of cases.

Some success has been achieved in inflammation arresting and regulation of indications for surgical treatment that produces positive results [10] [16]-[18]. Over the last several years, a number of reports related to new drugs for DAA symptomatic treatment and aortic wall strengthening have emerged.

The challenge of medical treatment is in the absence of clear ethiopathogenic presentation of DAA development and its management due to a leading role of atherosclerosis in this process, although 3\% - 7\% patients have no high BP or severe dyslipidemia. There have been no reports yet concerning clear predictors of aneurysm rupture as it may appear irrespectively of the severity of $\mathrm{AH}$, atherosclerosis or aneurysm diameter.

It is likely due to some occult factors present in DAA, for example, including solid (fibrosis) connective tissue dysfunction consisting of collagen and elastin contributing to a congenital fragility of aortic adventitious and medial frame function. An additional inflammatory process increases its destruction [11] [12] [14] [15].

Treatment of DAA is still considered a surgical issue, but a more in-depth look identifies in these individuals a number of other physical conditions, with the prevalence of cardiovascular disorders. The above-mentioned factors improve an already high mortality up to 32\% - 60\% [8] following aorta prosthetic operation.

Endovascular treatments of DAA patients can be an alternative to the conservative (surgical) modality. However, EVAR 1 and EVAR-2 studies showed that the number of fatal cases due to aneurysm rupture in conservatively treated patients was consistent with the number of ruptures, and post-operation mortality rate in endovascular treatment group. The absence of differences between key parameters may be explained, in authors' opinion, by a smaller frequency of aneurysm ruptures in patients who received conservative treatment [10] [18].

Sparing management for these patients has been offered earlier and it includes conservative and operative treatment provided only if there is a risk of ruptured aneurysm [16] [17] [19].

Objectives of the study. A comparison between a two and five-year survival in patients with atherosclerotic aneurysms of descending thoracic and abdominal aortic segments in the conservative treatment aimed at aortic wall strengthening and with surgical correction provided only if there is a risk its rupture.

\section{Materials and Methods}

\section{Patient Characteristics}

More than 240 individuals with descending thoracic and abdominal aortic aneurysms are now receiving treatment in Republican Research and Practical Centre "Cardiology". Among them, 121 patients entered a group of subjects who had participated in a 2-year study between 2011 and 2012, when the assessment was made of out- 
patient medical correction to improve aortic wall and control its further transformation with operative treatment offered only if there was a risk of ruptured aneurysm.

It allowed a clear approach to manage DAA patients based on conservative treatment modality. The aim was to attain and preserve optimal BP level, target lipid profile, lowered oxidative and inflammatory processes present in aorta, strengthening of aortic wall and stabilization of the condition. Dynamic follow-up of patients was made every 3 - 6 months. Well-being, aneurysm size, PB level, lipid profile, inflammatory process markers and signs of co-morbidities were recorded.

In 2014, statistical handling of data was made for 99 patients with a 4-year treatment. The results showed that over a 4-year period, no negative dynamics was present concerning aneurism size in patients with CRP $<2.7$ $\mathrm{mg} / \mathrm{l} ; \mathrm{LDL}<2.7 \mathrm{mmol} / \mathrm{l}$; atherogenic factor $<2.68 \mathrm{mmol} / \mathrm{l}$ and $\mathrm{BP}<132.4 / 78.6 \mathrm{~mm}$ Hg. This group was titled "the group of favorable course of the disease". In patients with negative dynamics in aneurysm size (by $>5 \mathrm{~mm}$ at 6 months), diameter at baseline was $>44.3 \mathrm{~mm}$; CRP > $8.3 \mathrm{mg} / \mathrm{l}$; LDL $>3.46 \mathrm{mmol} / \mathrm{l}$; AF $>4.05$ and $\mathrm{BP}>$ 142.1/86.77 mm Hg. These patients were included into "the group of unfavorable course of the disease" and received differentiated medical treatment with mandatory control every 3 months.

Indications for surgery are defined in case of ineffective treatment and failure to obtain target values of favorable course of the disease, and worsened baseline values of adverse course of DAA course.

The present study describes the causes of death defined in a group of 97 patients with 5-year treatment using self-developed methods.

\section{Methods}

1) Clinical investigations including CT angiography, aortic angiography, coronary angiography, US investigations.

2) Surgical treatment: aortic iliofemoral bifurcation prosthesis and endovascular stent-graft implantation.

3) Medical treatment aimed at aortic wall strengthening. Permanent treatment with the combination of ACE inhibitors with amlodipine, beta-blockers and statins. Treatment courses with macrolide antibiotics (roxithromycin, azithromycin) when signs of inflammatory process appear (by CRP data), and symptomatic treatment. C-reactive protein was defined in venous blood measured $\mathrm{mg} / \mathrm{l}$ using latex turbidimetric immunoassay by Cobas JNTEGRA 400 device by Roche Diagnostic test system (Switzerland).

4) Statistical analysis of the results was performed using Statistica 5.5 StatSoftCorp software (USA) for medical and biological investigations. Estimates of the studied parameters are presented as arithmetic mean value (M) and mean error of arithmetic mean value $(\mathrm{m})$. The Student $\mathrm{t}$-test was applied to define statistical significance. Differences obtained statistical significance when statistical significance of P value was above 0.05 .

\section{Results}

The groups of studied patients with DAA have not shown any statistically evident differences by age, sex, aneurysm diameter, including that of above and below $50 \mathrm{~mm}$, their length, diameters of thoracic and abdominal site, and by the ratio between the sites of descending thoracic aortic aneurysm and abdominal aortic aneurysm (Table 1). Statistical data were unaffected by the evidently decreased number of patients over a 5-year period.

Over a five-year period, 24 out of 97 patients received surgery (24.7\%) (see Table 2). Aortic prosthesis operation was performed in 16 patients, and eight patients were implanted with a stent-graft. Over the last year, no surgeries have been performed. All subjects received permanent medical treatment at doses consistent with the values for favorable and adverse courses of the disease.

Seventy-three subjects (75.3\%) received no surgery. It is noteworthy that this group included those with relatively small-sized aneurysms, elderly subjects with large aneurysms and a number of co-morbidities. The latter had absolute contraindications for surgical treatment. Therefore, mortality rates among patients treated conservatively was $32.9 \%$ at 5 years, and for those who underwent surgery it was $25 \%$.

Figure 1 shows that the highest mortality rate is due to comorbidities, especially in group of conservative treatment. This group comprised of7 elderly patients and those having large aneurysms, however, comorbidities were the main reason of death in the majority of cases. This cause was present in the group who received surgical treatment and made up $8.3 \%$.

The lowest 5-year mortality rate was associated with aneurysm rupture and was only $8.25 \%$. Moreover, it was the same for operated and non-operated subjects ( $8.3 \%$ and $8.22 \%$, respectively). Similar mortality (8.3\%) was 


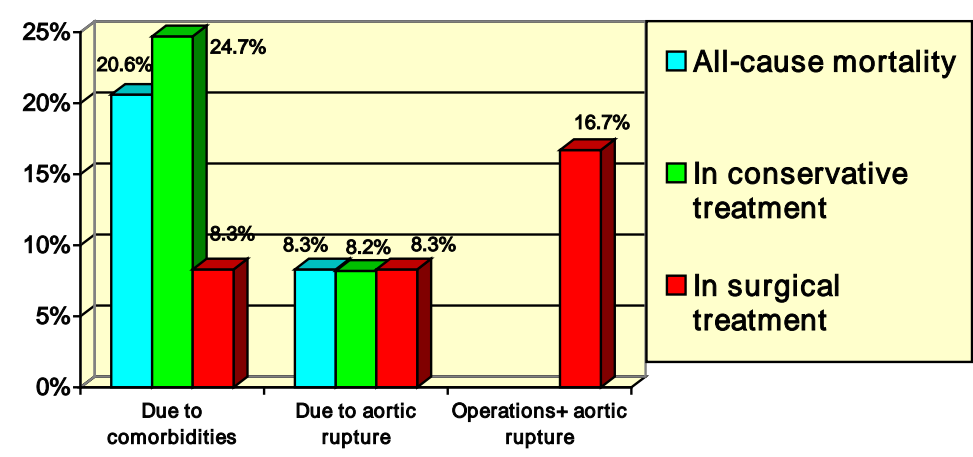

Figure 1. Main causes of mortality over a 5-year follow-up.

Table 1. Comparison between patients at 2.4 and 5 year-follow-up.

\begin{tabular}{|c|c|c|c|c|}
\hline Values & $\begin{array}{l}\text { Two-year follow-up } \\
\text { group } \mathrm{n}=121\end{array}$ & $\begin{array}{l}\text { Four-year follow-up } \\
\text { group } n=99\end{array}$ & $\begin{array}{l}\text { Five-year follow-up group } \\
\qquad \mathrm{n}=97\end{array}$ & $P$ \\
\hline Age, years & $67 \pm 0.77$ & $66.6 \pm 0.89$ & $66.6 \pm 0.9$ & \\
\hline M & $107 \%-88.4 \%$ & $86 \%-86.9 \%$ & $84 \%-86.6 \%$ & $>0.05$ \\
\hline $\mathrm{F}$ & $14 \%-11.6 \%$ & $13 \%-13.1 \%$ & $13 \%-13.4 \%$ & \\
\hline DAA & $91 \%-75.2 \%$ & $76 \%-76.8 \%$ & $75 \%-77.3 \%$ & $>0.05$ \\
\hline TDAA & $30 \%-24.8 \%$ & $23 \%-23.2 \%$ & $22 \%-22.7 \%$ & $>0.05$ \\
\hline d DAA, mm & $43.97 \pm 2.41$ & $44.1 \pm 1.7$ & $44.2 \pm 1.8$ & $>0.05$ \\
\hline d TDAA, mm & $48.88 \pm 2.64$ & $52.1 \pm 2.6$ & $52.2 \pm 2.5$ & $>0.05$ \\
\hline Patients with $\mathrm{d}<50 \mathrm{~mm}$ & $70 \%-57.8 \%$ & $66 \%-66.7 \%$ & $65 \%-67.0 \%$ & $>0.05$ \\
\hline Patients with $\mathrm{d}>50 \mathrm{~mm}$ & $51 \%-42.1 \%$ & $33 \%-34 \%$ & $32 \%-33.0 \%$ & $>0.05$ \\
\hline Aneurysm L, mm & $85.13 \pm 5.4$ & $86.97 \pm 5.1$ & $86.7 \pm 5.3$ & $>0.05$ \\
\hline
\end{tabular}

Table 2. Treatment outcomes in patients with descending atherosclerotic aneurysms at 5 years.

\begin{tabular}{|c|c|c|c|c|}
\hline Values & No surgery performed & Received surgery or stent-graft & $P$ & Total \\
\hline No. of patients & $73 \%-75.3 \%$ & $16+8=24 \%-24.7 \%$ & & $97 \%-100 \%$ \\
\hline Age (years at presentation) & $66.7 \pm 1.1$ & $64.1 \pm 1.9$ & $>0.05$ & $66.6 \pm 0.9$ \\
\hline Mortality rate by groups, common & $24 \%-32.9 \%$ & $4+2=6 \%-25 \%$ & $>0.05$ & $30 \%-30.9 \%$ \\
\hline -due to aortic rupture & $6 \%-8.22 \%$ & $1+1=2 \%-8.3 \%$ & $>0.05$ & $8 \%-8.25 \%$ \\
\hline -due to co-morbidities & $18 \%-24.7 \%$ & $1+1=2 \%-8.3 \%$ & $<0.05$ & $20 \%-20.6 \%$ \\
\hline -operation mortality & - & $2 \%-8.3 \%$ & & \\
\hline -mortality: aneurysm rupture + operation & $6 \%-8.2 \%$ & $4 \%-16.7 \%$ & $>0.05$ & $10 \%-10.3 \%$ \\
\hline
\end{tabular}

associated with the operation.

Despite the common opinion that aortic aneurysm is detrimental to patients' health, the 5-year follow-up show reverse results, i.e. the lowest mortality: aneurysm rupture occurred in only $8.25 \%$ of cases. Moreover, the estimates were similar to the estimates in non-operated patients.

Consistent mortality (8.3\%) was related to operative treatment. It is noteworthy, that surgical treatment is aimed at aneurysm rupture and early death prevention. Therefore, it seems feasible to consider cases of post-operation mortality and aortic ruptures together. They made up $16.7 \%$ which is slightly inferior to mortality due to co-morbidities.

Mortality dynamics at 2.4 and 5 years is shown in Figure 1 and Figure 2. It reflects the mortality changes seen in conservative vs. surgical treatment. It allows for an assessment of mortality changes in conservative and 


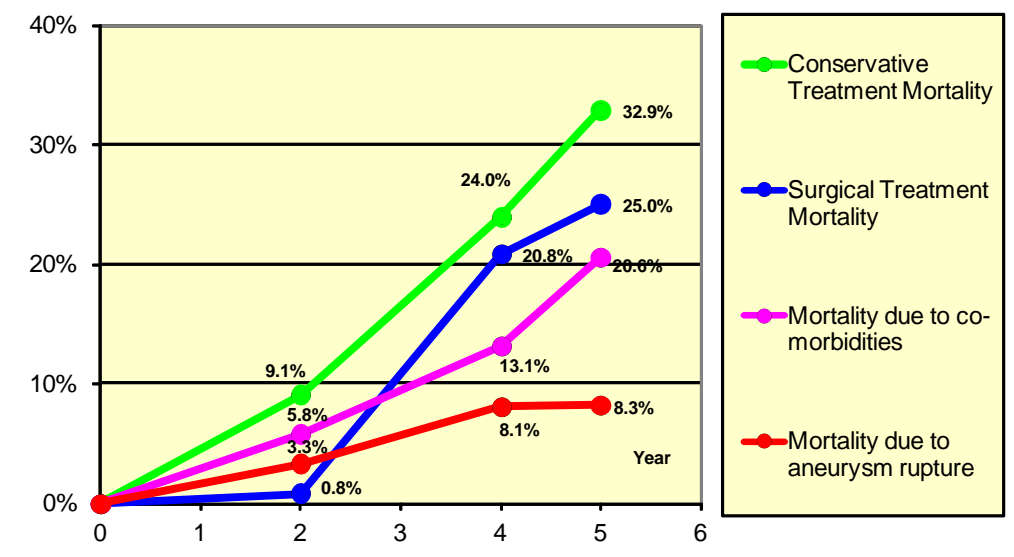

Figure 2. Mortality dynamics in descending aortic aneurysms at 2, 4 and 5 years.

surgical treatment in the follow-up period. Figure 1 shows that mortality rates due to co-morbidity over the abovementioned period tended to increase in ascending order. A curve for aneurysm rupture rises horizontally for 4 years and remains at rest at 5 years.

Mortality curve in conservatively treated group increases with acceleration through the years. Mortality curve in surgically treated group rose slightly in the first 2 years with a steep rise observed in subsequent years.

Additionally, the analysis of mortality related to aneurysm diameter was performed. The results showed that more than half of deaths among non-operated patients over 5 years (14 cases) were registered in patients with aneurysm diameter $>50 \mathrm{~mm}$, but aneurysm rupture was seen in less than half of patients (6 cases). Other (7 cases) patients died from comorbidities. All these patients had counter indications to surgery due to the severity of aortic lesion or present fatal concurrent disease and complications.

When the aneurysm diameter is below $50 \mathrm{~mm}$, death is related to the concomitant condition. Although there were no cases of aneurysm rupture among them, still there were two such cases at 5 years occurred in patients with the aneurysm diameter of 37 and $42 \mathrm{~mm}$. The first patient experienced rupture at presentation, and doctors could not save him. The second patient developed pain syndrome on the next day after the examination, and he received emergency aortic prosthesis. These patients were excluded from the testing program, as they had not been examined and received no targeted treatment. It was concluded that the small number of aneurysm ruptures seen in our groups was associated with medical treatment to prevent these cases.

In the group of 24 operated patients over a 5 year-period 6 patients (25\%) died: two due to a co-morbidity, and two due to aneurysm rupture, and two more following operation. Although this group contained relatively safe patients that were almost consistent with those having aneurysm below $50 \mathrm{~mm}$, still the mortality rate was higher.

\section{Discussion}

The discussed groups of patients with DAA were similar by key characteristics, i.e. sex, age, aortic lesion and diameter as they had similar condition and settings. This similarity was observed during a five-year period and is reflected in mortality estimates. Co-morbidities contribute a great deal in fatal cases. With aging, patients develop signs of various diseases that become literally fateful. This factor was more evident in the group of conservatively treated patients because it comprises most severely affected patients. The similar process, although to a lesser extent, affects the group of surgical treatment contributing to a fatal outcome in patients who recently received surgery due to aneurysm.

Figure 1 shows that mortality dynamics due to aneurysm rupture does not fully fit into the dynamics of other deaths. Nonetheless, the main trouble with DAA is first of all aneurysm rupture, which is the basis on which we build the whole strategy of operative treatment. However, over a 5-year follow-up, there were only eight aneurysm ruptures out of 97 cases with no significant differences between operated and non-operated individuals. It challenges feasibility of current treatment modalities to treat DAA.

Over a 5-year period, there were no cases of aneurysm rupture. It can be explained by the fact that in the first 2 years, our approaches to management of DAA subjects had not been perfect and we could make mistakes as to the intricacies of medical procedures. It deals largely with CRP standard and compliance. These factors contributed 
mostly to aneurysm ruptures and defined patient's future. In patients with previous aneurysm rupture CRP was $18.0 \pm 7.9 \mathrm{mg} / \mathrm{l}$ at baseline, whereas in other patients, it was $8.7 \pm 4.1 \mathrm{mg} / \mathrm{l}$.

It suggests that the inflammatory process may be a key factor contributing to the aneurysm progression. Statistical CRP estimate of $2.7 \mathrm{mg} / \mathrm{l}$ in favorable course strongly suggests that if the value exceeds $3 \mathrm{mg} / \mathrm{l} \mathrm{mark,} \mathrm{a}$ doctor should consider this factor as contributing to the progression of the disease.

Despite the fact that new mechanisms of DAA formation have been now fully understood and the necessary experience of treatment has been accumulated, still we are not always able to find effective correction methods. This is not about surgical approach that is essentially urgent. Inflammatory process suppression, BP control and aneurysm wall strengthening are thought to be not enough to obtained positive results. Trust relationship between a doctor and patient is what comes first. Otherwise, our efforts will be doomed to failure. Thus, 75\% of fatal cases because of aneurysm rupture were due to an extremely low compliance.

Most of published DAA data suggest that the more severe course is observed in thoracic (descending) localization. Our data show the opposite: 5-year all-cause mortality was $18.2 \%$ in descending thoracic aortic aneurysm, 34.7\% in abdominal aortic aneurysm; $9.1 \%$ and $8 \%$ due to aneurysm rupture; $9.1 \%$ and $26.7 \%$, respectively due to comorbidities. From our perspective, these specific presentation of abdominal aortic aneurysm is related to visceral organ and renal chronic ischemia.

\section{Conclusions}

Not fully satisfactory results of surgical treatment of descending thoracic and abdominal aortic atherosclerotic aneurysms necessitated detailing of origin, pathogenesis and management for these patients. Experimental [13] [20] and clinical investigations [10] [16]-[19] have shown possibility and feasibility of medical treatment of the condition in the majority of patients. However, it was needed to alter indications to surgical treatment, as aneurysm progression dynamics does not always reflect the severity of the condition. Inflammatory process, comorbidities and compliance play a crucial role.

Our five-year investigations of patients with DAA [16] [17] [19] have shown that the causes of the negative curse of the disease are multifactorial and related to destructive processes taking place in aortic wall, its inflammation, impaired lipid exchange, arterial hypertension and low compliance. This condition emerges when baseline aneurysm diameter is $>44.3 \mathrm{~mm}$; CRP > $8.3 \mathrm{mg} / \mathrm{l}$; LDL $>3.46 \mathrm{mmol} / \mathrm{l}$; AF $>4.05$. BP $>142.1 / 86.77 \mathrm{mmHg}$, and compliance is poor. These patients are referred to the group of risk of adverse course in which we use intense differentiated medical treatment followed by mandatory control of treatment efficacy and trends every 3 months.

Assertive treatment to correct the abovementioned parameters contributes to stabilization in most of the patients. In case when conservative approach is inefficient and a poor dynamics is observed, the only way is to open prosthesis of aorta and stent-graft implantation. In this situation, aneurysm diameter is not a key factor in determining the indications for operation, as it is highly dependent on the rest of the factors already mentioned suggesting a possible aneurysm changes from process stabilization to the wall lost resistance to rupture.

This approach is consistent with investigations EVAR 1 and EVAR-2 [10] [18], which showed that the number of deaths due to aneurysm rupture in conservatively treated group was consistent with the number of aneurysm ruptures and postoperation mortality in endovascular treatment group.

The experience of sparing treatment of DAA individuals has shown that aneurysm rupture as the cause of death in patients with DAA is at a lower position (8.3\%) and completely corresponds as a percentage to deaths after operation (16.7\%). Thus, speculative survival is $91.7 \%$ in conservative treatment, and $83.3 \%$ in surgical treatment which is inferior to surgical approach.

The main cause of death in DAA was comorbidity (20.6\%). Therefore, special emphasis is to be made on this to define treatment modalities for these patients. Considering this, a 5-year survival rate in sparing treatment was $69.1 \%$ for all-cause mortality, $67.1 \%$ for conservative treatment, and $75 \%$ in patients who underwent surgery. There were no evident differences between these estimates. A mild difference between the survivors can be explained by the fact that patients who received surgical treatment had had fewer comorbidities which were largely anticipated with a successful surgery.

Based on literature data, survival is scattered. Data specified in the introduction (6\% - 50\%) about the patients who survived over a 5-year follow-up period is largely inconsistent with our results. We are convinced that the DAA operative intervention is better to perform after a long-term conservative repair of dyslipidemia, arrest of 
inflammatory process (if present), control of adequate BP levels and compliance to treatment, and thorough monitoring of concomitant diseases. Operative treatment should not be the aim in itself but the result of the dynamics of the disease and assurance in positive surgery outcomes.

Additionally, the sparing management implicates cost-effectiveness due to a reduced number of unjustified operations. Taken together, it allows recommending the sparing option for patients with DAA as the treatment of choice.

\section{References}

[1] Shirinbek, O. and Bakulev, A.N. (2008) Infrarenal Abdominal Aneurisms: Modalities and Outcomes (Literature Review). Cardiovascular Disease Journal, RAMN (Russian Academy of Medical Sciences), 9, 50-57. (in Russian)

[2] Hallett Jr., J.W. (1992) Abdominal Aortic Aneurysm: Natural History and Treatment. Heart Disease and Stroke, 1, 303-308.

[3] Bokeria, L.A., Arakelyan, V.S. and Bakulev, A.N. (2010) Aortic Segment Thoracic and Thoracic Abdominal Aneurism Surgery: Guidelines for Practitioners. RAMN (Russian Academy of Medical Sciences), 394. (In Russian)

[4] ESC Guidelines (Committee for Practice Guidelines to Improve the Quality of Clinical Practice and Patient Care in Europe). www.escardio.by

[5] Restrepo, C.S., Jcazionez, D., Suri, R. and Vargas, D. (2011) Aortitis: Imaging Spectrum of the Infectious and Inflammatory Conditions of the Aorta. Radiographics, 31, 435-451.

[6] Harrington, D.J. (1996) Bacterial Collagenases and Collagen-Degrading Enzymes and Their Potential Role in Human Disease. Infection and Immunity, 64, 1885-1891.

[7] Milewicz, D.M. (2012) MicroRNAs, Fibrotic Remodeling, and Aortic Aneurysm. Journal Clinical Investigation, No. 2, 490-403.

[8] Boersma, E., Kertai, M.D., Schouten, O., et al. (2005) Perioperative Cardiovascular Mortality in Noncardiac Surgery: Validation of the Lee Cardiac Risk Index. The American Journal of Medicine, 118, 1134-1141. http://dx.doi.org/10.1016/j.amjmed.2005.01.064

[9] Greenhalgh, R.M., Brown, L.C., Kwong, G.P., et al. (2004) EVAR Trial Participants. Comparison of Endovascular Aneurysm Repair with Open Repair in Patients with Abdominal Aortic Aneurysm (EVAR Trial 1), 30-Day Operative Mortality Results: Randomized Controlled Trial. The Lancet, 364, 843-848.

[10] EVAR Trial Participants (2005) Endovascular Aneurysm Repair versus Open Repair in Patients with Abdominal Aortic Aneurysm (EVAR Trial 1): Randomized Controlled Trial. The Lancet, 365, 2179-2186.

[11] John, K.A., Lusher Thomas, F. and Serruys Patrick, W. (2011) Cardiovascular Diseases. ECS Guidelines. “GEOTARMedia”, C, 1209-1248. (Translated into Russian)

[12] Jeffrey, A.J. Spinale, F.G. and Ikonomidis, J.S. (2009) Transforming Growth Factor-Beta Signaling in Thoracic Aortic Aneurysm Development: A Paradox in Pathogenesis. Journal of Vascular Research, 46, 19-37.

[13] Yoshimura, K. and Aoki, H. (2012) Recent Advances in Pharmacotherapy Development for Abdominal Aortic Aneurysm. International Journal of Vascular Medicine, 2012, 648167.

[14] Mikhaylov, D.V., Vinokurov, I.A., Bogopolskaya, O.M., et al. (2012) Perspectives of Preventive Treatment of Large Vessel Aneurysms. Kardiologiya i sedechnososudistaya khirurgiya, 4, 51-56.

[15] Cherepakhin, D.I., Bazilev, V.V., Evtyushkin, I.L., et al. (2012) Large Vessel Aneurysms in the Era of Genomics and Proteomics and Prognostic Medicine Options. Kardiologiya i sedechnososudistaya khirurgiya, 4, 58-62.

[16] Krylov, V.P., Mrochek, A.G., Titov, L.P., Gaiduk, V.N., Reut, L.I. and Smaliakou, A.L. (2014) Can We Change a Look at Atherosclerotic Aortic Aneurism Treatment. Health, 6, 1345-1351. http://dx.doi.org/10.4236/health.2014.612165

[17] Krylov, V.P., Titov, L.P., Gayduk, V.N., Reut, L.I., Smaliakou, A.L. and Mankevich, N.V. (2015) Atherosclerotic Descending Aortic Aneurysm. Pros and Cons of Surgery. World Journal of Cardiovascular Surgery, 5, 91-101. http://dx.doi.org/10.4236/wjcs.2015.59015

[18] EVAR Trial Participants (2005) Endovascular Aneurysm Repair and Outcome in Patients Unfit for Open Repair of Abdominal Aortic Aneurysm (EVAR Trial 2): Randomized Controlled Trial. The Lancet, 365, 2187-2192.

[19] Mankevich, N.V., Mrochek, A.G., Krilov, V.P., Gayuduk, V.N., Reut, L.I. and Smoliakou, A.L. (2015) Predictors of Progression of Atherosclerotic Descending Aortic Aneurysm and Correction. Kardiologiya v Belarusi, 5, 63-73.

[20] Yoshimura, K., Aoki, H., Ikeda, Y., Furutani, A., Hamano, K. and Matsuzaki, M. (2006) Regression of Abdominal Aortic Aneurysm by Inhibition of c-Jun N-Terminal Kinase in Mice. Annals of the New York Academy of Sciences, 1085, 74-81. 


\section{Abbreviations}

DTAA — descending thoracic aortic aneurysm

AAA — abdominal aortic aneurysm

DAA — descending aortic aneurysm

US-ultrasonography

CT-computer tomography

$\mathrm{CRP}$-C-reactive protein

LDL-low-density lipoprotein

$\mathrm{AI}$-atherogenic index

BP-blood pressure

$\mathrm{d}$-aneurysm diameter in $\mathrm{mm}$

l-aneurysm length in $\mathrm{mm}$ 